\title{
Object Recognition and Tracking in Video Sequences: A New Integrated Methodology
}

\author{
Nicolás Amézquita Gómez ${ }^{1}$, René Alquézar ${ }^{2}$, and Francesc Serratosa ${ }^{1}$ \\ ${ }^{1}$ Departament d'Enginyeria Informàtica i Matemàtiques, Universitat Rovira i Virgili, \\ Campus Sescelades, Av. dels Països Catalans 26, 43007, Tarragona, Spain \\ ${ }^{2}$ Dept. Llenguatges i Sistemes Informàtics, Universitat Politècnica de Catalunya, \\ Campus Nord, Edifici Omega, 08034 Barcelona, Spain \\ \{francesc.serratosa, nicolas.amezquita\} aurv.cat, \\ \{amezquita, alquezar\}@lsi.upc.edu
}

\begin{abstract}
This paper describes a methodology that integrates recognition and segmentation, simultaneously with image tracking in a cooperative manner, for recognition of objects (or parts of them) in image sequences. A probabilistic general approach at pixel level is depicted together with a practical heuristic simplification in which pixels' class probabilities are approximated by a finite small set of class possibility values. These possibility values are updated iteratively along the image sequence for each class and each pixel taking into account both the prior tracking information and the spot-based object recognition results provided by a trained neural network. A further segmentation of the class possibility images allows the tracking of each object of interest in the sequence. The good experimental results obtained so far show the viability of the approach under certain conditions.
\end{abstract}

Keywords: Object recognition, object tracking, image segmentation, neural networks, probabilistic approach, video sequences.

\section{Introduction}

This work presents a methodology that integrates segmentation, recognition and tracking, for recognition of objects in image sequences. To the best of our knowledge there are few existing works that combine segmentation, recognition and tracking in an integrated framework [1]. These tasks often are treated separately and/or sequentially on intermediate representations obtained by the segmentation and grouping algorithms [2, 3, 4]. In [5], object recognition techniques are applied to a scene where the objects of interest do not move most of the time and makes tracking a discrete process of watching for object disappearances and reappearances.

The procedure that we used is based on the iterative and adaptive processing of consecutive frames. A similar methodology is presented in [6]. Another related work is [7], where a probabilistic approach that combines segmentation, object recognition, 3D localization and tracking in an integrated and unified framework is described. In our case, the original images are firstly segmented in homogeneous regions (spots) and color and geometric features are extracted from these regions. As reported in [8], neural networks can be trained to classify spots into different objects using the spot 
features as input, provided that an enough large set of labeled spots is given from the supervised segmentation of representative views of these objects. In [8], the trained networks were shown to classify quite correctly test spots located in the same regions of interest that the training spots (ROI that were defined around each object). However, the spot classification performance impairs significantly outside these regions or in different images than those used for training.

In the current work, we address this problem (object recognition in full new images) through the use of a dynamic iterative approach in which a probabilistic model at pixel level (or an approximation of it) is updated taking into account both the neural net outputs and prior object tracking information from the previous image.

A scheme of the whole process integrating object recognition and tracking is displayed in Fig.1.

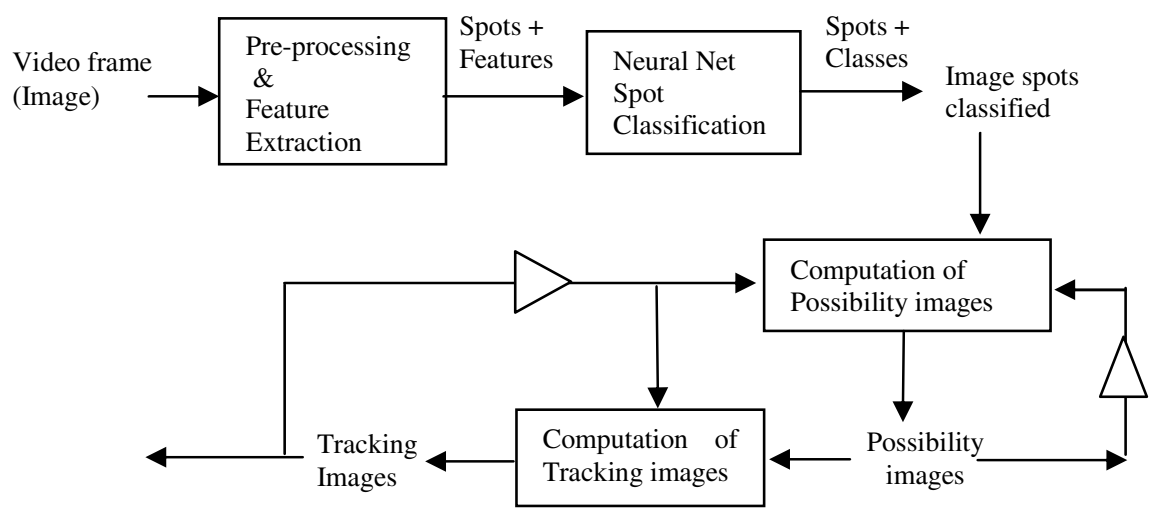

Fig. 1. Block diagram of the iterative object recognition and tracking process

The rest of the paper is organized as follows. A more formal definition of the addressed problem is given in Section 2, together with the entire notation used throughout the paper. In Section 3, the proposed methodology is described in more detail. Experimental results are included in Section 4 and, finally, conclusions and future work are discussed in Section 5.

\section{Problem Statement and Notation}

Let us assume that we have a sequence of 2D color images $I^{t}(x, y)$ for $t=1, \ldots, L$, and a corresponding sequence $S^{t}(x, y)$ of segmented images resulting from the application of an image segmentation algorithm to the former. Also, let us consider that there are (or can be) $N$ objects of interest in the sequence of different types (associated with classes $c=1, \ldots, N)$, and that a special class $c=N+1$ is reserved for the background. Furthermore, let us assume that the initial position of each object is known and represented by $N$ binary images, $p_{c}{ }^{0}(x, y)$, for $c=1, \ldots, N$, where $p_{c}^{0}(x, y)=1$ means that the pixel $(x, y)$ belongs to a region covered by an object of class $c$ in the first image. 
We would like to obtain $N$ sequences of binary images $T_{c}^{t}(x, y)$, for $c=1, \ldots, N$, that mark the pixels belonging to each object in each image; these images are the desired output of the whole process and can also be regarded as the output of a tracking process for each object. Note that we can initialize these tracking images (for $t=0$ ) from the given initial positions of each object, this is:

$$
T_{c}^{0}(x, y)=p_{c}^{0}(x, y)
$$

For notational purposes, let $M C{ }_{c}^{t}$, for $c=1, \ldots, N$, refer to the mass centers of each object in the corresponding tracking image $T_{c}{ }_{c}^{t}(x, y)$.

Suppose that a neural network has been trained to classify regions (spots) of the same objects using a different but similar sequence of labeled segmented images. Hence, the trained network is able to produce a sequence of class probability images $Q_{c}{ }^{t}(x, y)$ for $t=1, \ldots, L$ and $c=1, \ldots, N+1$, where the value $Q_{c}{ }^{t}(x, y)$ represents the aposteriori probability given by the net output that the pixel $(x, y)$ of the segmented image $S^{t}(x, y)$ belongs to the class $c$. From these probabilities, a class can be assigned to each pixel simply by choosing the class with maximum probability:

$$
C^{t}(x, y)=\underset{c}{\arg \max }\left(Q_{c}^{t}(x, y)\right)
$$

In order to obtain the tracking images, a probabilistic approach could be followed in which we would need to store and update $N+1$ probability images $p_{c}^{t}(x, y)$, for $c=1, \ldots, N+1$, where the value $p_{c}{ }_{c}^{t}(x, y)$ represents the probability that the pixel $(x, y)$ in time $t$ belongs to an object of class $c$ (for $c=1, \ldots, N$ ) or to the background (for $c=N+1$ ). In general, these probabilities should be computed as a certain function $f$ of the same probabilities in the previous step, the class probabilities given by the neural net for the current step and the tracking images resulting from the previous step:

$$
p_{c}^{t}(x, y)=f\left(p^{t-1}(x, y), Q^{t}(x, y), T^{t-1}(x, y)\right)
$$

Now, the tracking images could be computed dynamically using these probabilities according to some decision function $d$ :

$$
T_{c}^{t}(x, y)=d\left(p^{t}(x, y), T_{c}^{t-1}(x, y)\right)
$$

In the present work, as a first simple approach to test, we have relaxed the normalization constraint required for probabilities and have approximated the probability values with a small set of "possibility" values (e.g. 0, 1/2,1) computed heuristically. Hence, instead of using the probability images $p_{c}^{t}(x, y)$ we have used the so-called "possibility" images $H_{c}^{t}(x, y)$, that contain the possibility values that a pixel $(x, y)$ belongs to a class $c$ in time $t$. Note that these images can be initialized as well from the given initial positions of each object:

$$
H_{c}^{0}(x, y)=p_{c}^{0}(x, y)
$$

Consequently, the update function $f$ and the decision function $d$ have been defined in this work using the possibility images $H_{c}^{t}(x, y)$ instead of the probability images $p_{c}{ }^{t}(x, y)$, in the way described in next section. In particular, the class assignments given 
by the net $C^{t}(x, y)$ have been used instead of the probabilities $Q_{c}{ }^{t}(x, y)$ in the update function $f$.

\section{Methodology}

The methodology proposed can be split in two phases: the object learning phase and the object recognition and tracking phase. Next subsections describe both phases.

\subsection{Object Learning}

For object learning, a sequence of segmented images showing the objects of interest is required. Furthermore, a subset of the spots (segmentation regions) obtained must be selected and labeled manually (or semi-automatically as described in [8]) with the target classes. These target classes include the different object types and a special class for the background. In addition, for each selected spot, a number of features have to be computed that may include both color and geometric properties.

The spot features and target classes are collected in a pattern file. Then, a neural network is trained to classify the selected spots using most of the patterns as training set and the rest as validation set. Once trained, when a new pattern (spot feature vector) is introduced, the network is able to estimate the a-posteriori class probabilities for this pattern, according to the statistical model it has learnt previously from the given examples. From these probabilities, a class can be assigned to each spot simply by choosing the class with maximum probability. Note that if we represent the probabilities and classes at pixel level rather than at spot level, all pixels of a given spot will have the same probabilities and class that the entire spot.

A more detailed description of the learning phase that includes the specific features used for the spots is available in [8].

\subsection{Object Recognition and Tracking}

For object recognition and tracking, another sequence of segmented images showing the same objects of interest is required. Furthermore, for each object of interest, its approximate location in the first image of the sequence is needed. This information is supposed to come as a binary image for each object, where the white pixels represent the object and the black pixels represent the background or other objects. These binary images are used to set the initial values of both the tracking images and the possibility images, as defined in Section 2, equations (1) and (5). For the following time steps $t=1,2, \ldots L$, the binary images that represent the approximate positions of the objects of interest (tracking images) will be computed as explained later.

The neural network obtained in the learning phase is applied to all the spots of all the images in the recognition sequence. This means that all the spot features must be computed previously. From the network outputs, all spots (and their constituent pixels) can be classified according to equation (2).

In order to update the tracking images, first a possibility image is computed for each class and time step. The update function $f$ for the possibility image $H_{c}^{t}(x, y)$ is defined heuristically taking into account the classification of pixel $(x, y)$ given by the 
neural network, $C^{t}(x, y)$, and the previous values of the pixel in the tracking image $T_{c}^{t-1}(x, y)$ and the possibility image $H_{c}^{t-1}(x, y)$. Specifically, we used as function $f$ the mapping shown in Table 1.

Table 1. Update function for the possibility image $H_{c}^{t}(x, y)$. The two shadowed entries correspond to impossible cases, since $T_{c}^{t-1}(x, y)=1 \Rightarrow H_{c}^{t-1} \geq 1 / 2$.

\begin{tabular}{lll|l}
\hline $\mathrm{T}_{\mathrm{c}}{ }^{\mathrm{t}-1}$ & $\mathrm{H}_{\mathrm{c}}{ }^{\mathrm{t}-1}$ & $\mathrm{C}_{\mathrm{c}}{ }^{\mathrm{t}}=\mathrm{c}$ & $\mathrm{H}_{\mathrm{c}}{ }^{\mathrm{t}}$ \\
\hline 0 & 0 & No & 0 \\
0 & $1 / 2$ & No & 0 \\
0 & 1 & No & 0 \\
0 & 0 & Yes & $1 / 2$ \\
0 & $1 / 2$ & Yes & $1 / 2$ \\
0 & 1 & Yes & $1 / 2$ \\
1 & 0 & No & 0 \\
1 & $1 / 2$ & No & 0 \\
1 & 1 & No & $1 / 2$ \\
1 & 0 & Yes & $1 / 2$ \\
1 & $1 / 2$ & Yes & 1 \\
1 & 1 & Yes & 1 \\
\hline
\end{tabular}

Then, this possibility image $H_{c}^{t}(x, y)$ is segmented inside a region-of-interest ROI $_{c}^{t}$, which is estimated from the bounding box $B B_{c}^{t-l}$ of the previous tracking image $T_{c}^{t-1}$. In order to compute the bounding box of a binary image, such as $T_{c}^{t-I}$, we use the method described in [9]. In fact, $R O I_{c}{ }^{t}$ and $B B_{c}^{t-1}$ share the same center and shape, but the size of region $\mathrm{ROI}_{c}{ }^{t}$ is determined to be greater than that of $B B_{c}{ }^{t-1}$ according to a given fixed scale ratio $r$ (e.g. $r=1.25$ ) to take into account a possible displacement of

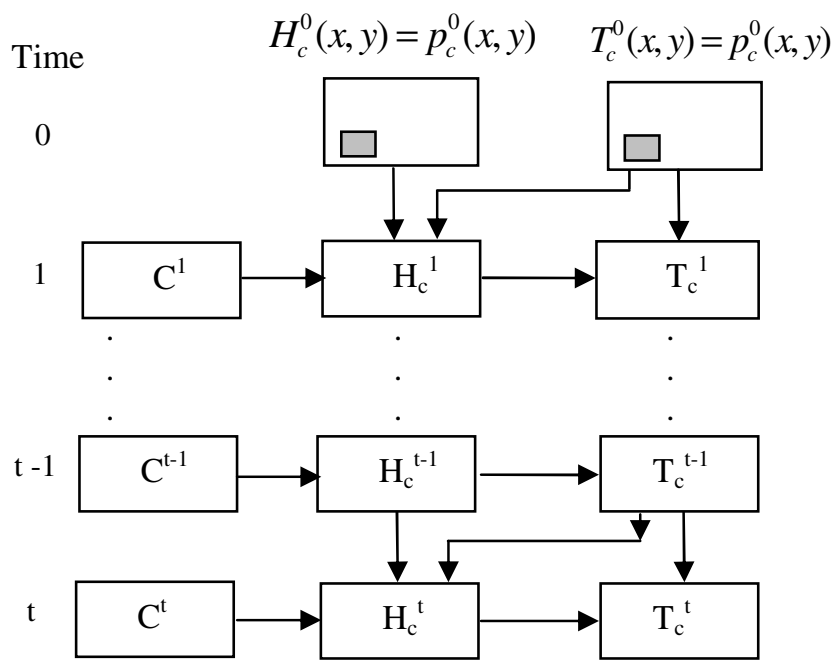

Fig. 2. Dynamic calculation of $H_{c}^{t}(x, y)$ and $T_{c}^{t}(x, y)$ 
the object between consecutive frames. The region of interest $R_{O I}{ }_{c}^{t}$ is then passed to a seed-based segmentation algorithm [10] that yields the next tracking image $T_{c}^{t}$ by finding a single connected region of the image $H_{c}^{t}(x, y)$, within the limits of $R O I_{c}{ }_{c}^{t}$, such that all their pixels have a possibility value $\geq$ than a threshold $z$ (e.g. $z=1 / 2)$, where the seed pixel is defined as $M C_{c}^{t-1}$, the mass center of $T_{c}^{t-1}$.

Summarizing, for the each time step $t$ the next processes are carried out sequentially:

1. Calculate the class assignment $C^{t}(x, y)$ from the outputs given by the neural network when the features of the spot that includes the pixel $(x, y)$ are entered to the net.

2. Compute $H_{c}^{t}(x, y)$ from $H_{c}^{t-1}(x, y), T_{c}^{t-1}(x, y)$ and $C^{t}$, for each class $c=1, \ldots, N$, using the heuristic mapping defined in Table 1 .

3. Calculate $T_{c}^{t}(x, y)$ from $H_{c}^{t}(x, y)$ and $T_{c}^{t-1}(x, y)$, for each class $c=1, \ldots, N$, by finding the region of interest $\mathrm{ROI}_{c}{ }^{t}$ and applying within it the seed segmentation algorithm to $H_{c}^{t}(x, y)$.

These steps are shown graphically in figure 2 for a sequence of time steps.

\section{Experimental Results}

We illustrate our methodology and approach using two sequences of images that correspond to the left and right image sequences of a stereo vision system installed on a mobile robot. These sequences display an indoor scene where we chose three objects of interest $(N=3)$ : a box, a chair and a pair of adjacent wastebaskets. The slow relative motion of the objects in the sequences is due to the slow motion of the mobile robot during its navigation in an indoor environment, and this small displacement of the objects is an expected characteristic of the video sequences we are dealing with. In our initial work described in [8], only the left sequence was used and only the spots inside some predefined ROIs were selected for neural network training and test; a cross-validation procedure was followed using $25 \%$ of the spots for testing with a correct classification performance of around $76 \%$. In a more recent work [11], this performance was raised to a $96 \%$ by adjusting more accurately the ROIs and to a $99 \%$ by combining the neural net with a reclassification process based on clustering. The performance of the selected neural net on the right sequence was a $90 \%$ of correctly classified patterns in the same ROIs.

However, for the test phase, it is somewhat tricky to restrict the object recognition to predefined ROIs, since we cannot rely on having the ROIs marked on every frame in a realistic experimental scenario. Hence, in the new experiments reported here, the same neural network trained from selected ROIs in the left sequence was used, but the whole right sequence including all spots was taken for testing both object recognition and tracking. A ROI for each object was only defined in the first image to initialize the tracking images. To the contrary of the results in [8] and [11], in this work we were not so interested in achieving a high spot classification ratio but a sequence of tracking images of good quality for each object of interest, as a first validation of the methodology proposed in Section 3. 

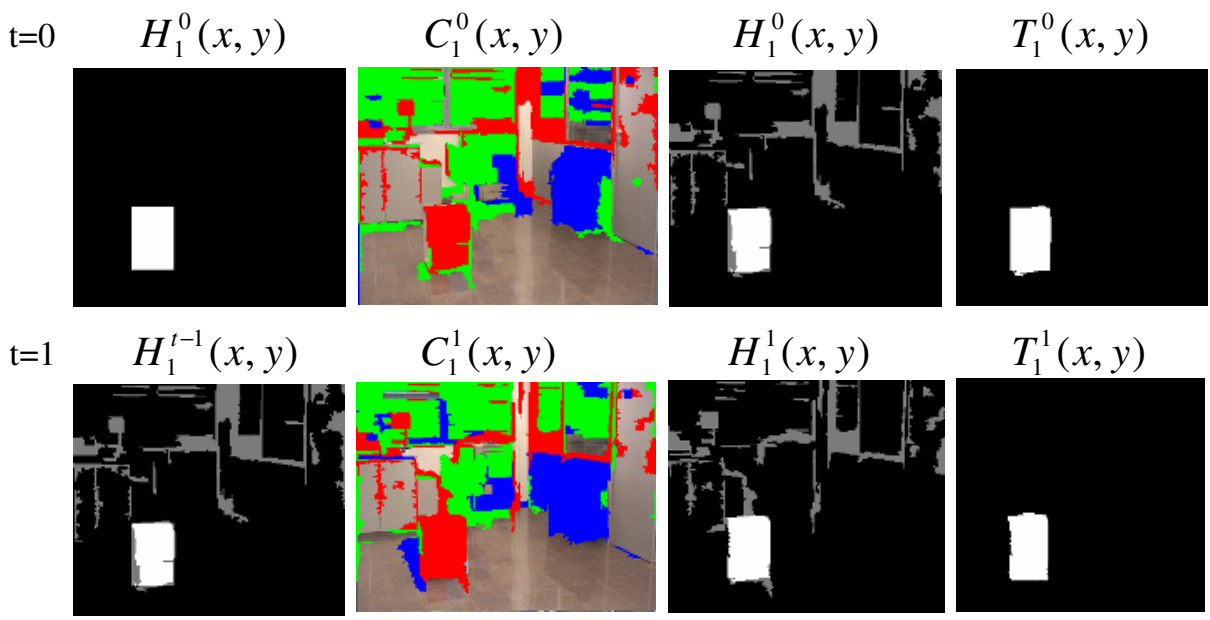

$$
\mathrm{t}=2 \quad H_{1}^{t-1}(x, y)
$$

$$
C_{1}^{2}(x, y)
$$

$$
H_{1}^{2}(x, y)
$$

$$
T_{1}^{2}(x, y)
$$
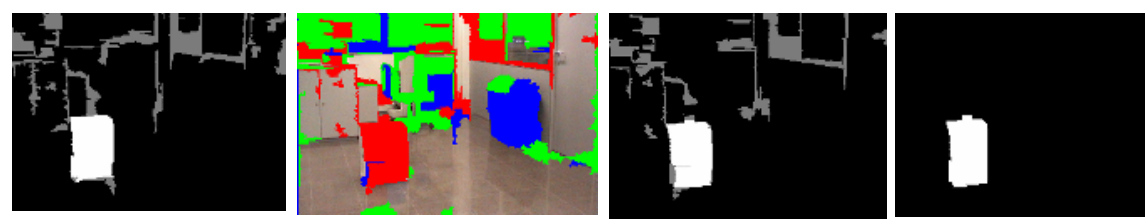

$\mathrm{t}=3$

$$
H_{1}^{t-1}(x, y)
$$
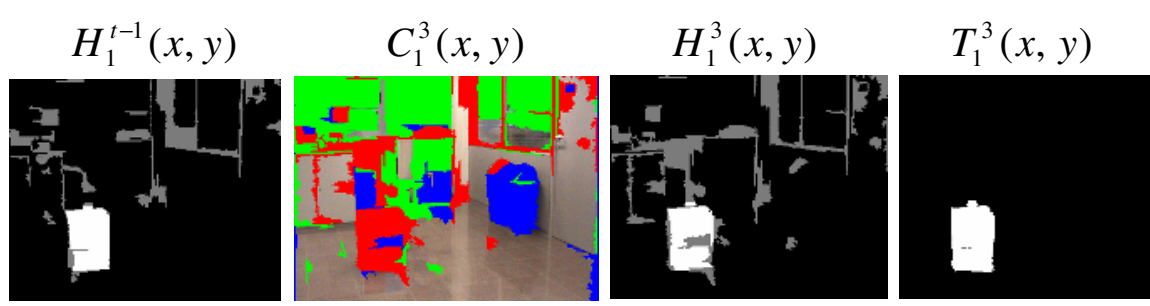

Fig. 3. Recognition and tracking results for class 1 (the box) in the first 4 frames of the test sequence

Figures 3 and 4 illustrate the process depicted in Figure 2 for two of the objects of interest (the box and the wastebaskets, respectively) in some consecutive images of the test sequence. Using the obtained tracking binary images as a visualization mask, the results of tracking both objects on the original images are displayed in Figures 5 and 6 . It can be observed that the proposed approach obtained rather satisfactory results on these images. Similar good results were obtained for these two objects in the rest of the sequence, but those for the other object (the chair) were not so stable. Concerning the computation time of our current non-optimized implementation, the process of recognition and tracking takes about 20 seconds in average for each object and frame in a Pentium IV processor at $3.4 \mathrm{GHz}$ with 512 MB RAM. 

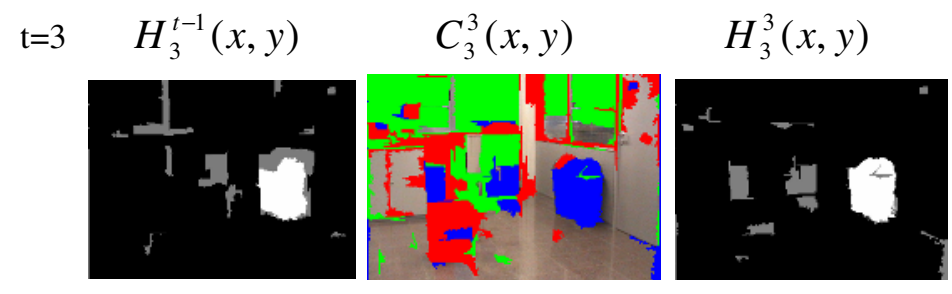

$$
T_{3}^{3}(x, y)
$$

$\mathrm{t}=4 \quad H_{3}^{t-1}(x, y)$

$C_{3}^{4}(x, y)$

$H_{3}^{4}(x, y)$
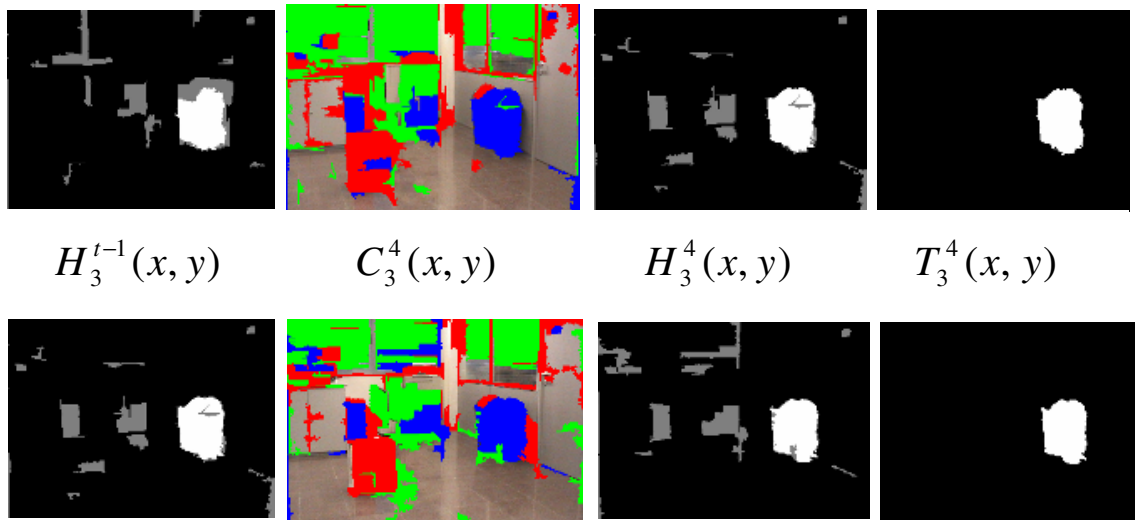

$T_{3}^{4}(x, y)$

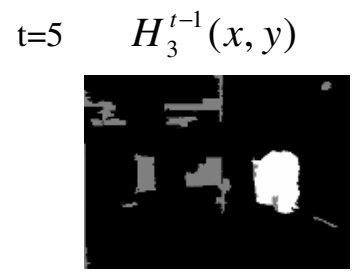

$C_{3}^{5}(x, y)$

$$
H_{3}^{5}(x, y)
$$

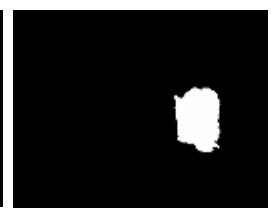

$\mathrm{t}=6$
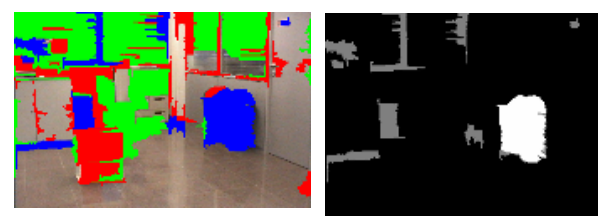

$$
T_{3}^{5}(x, y)
$$

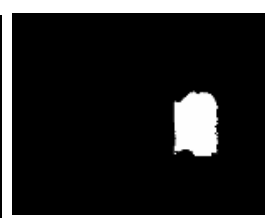

$$
H_{3}^{t-1}(x, y) \quad C_{3}^{6}(x, y)
$$

$H_{3}^{6}(x, y)$

$T_{3}^{6}(x, y)$
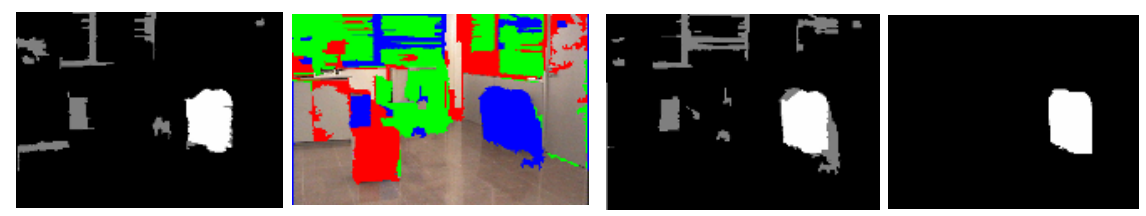

Fig. 4. Recognition and tracking results for class 3 (the wastebaskets) in four frames of the test sequence

Frame 1592

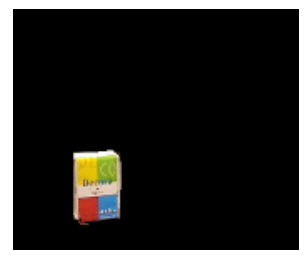

Frame 1593

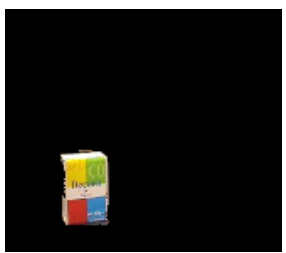

Frame 1594

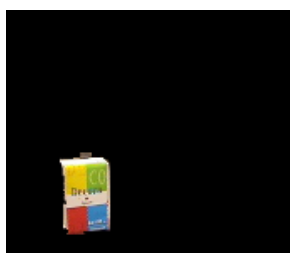

Frame 1595

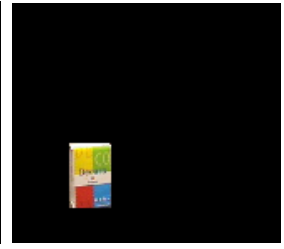

Fig. 5. Tracking of the box on part of the original image sequence 
Frame 1595

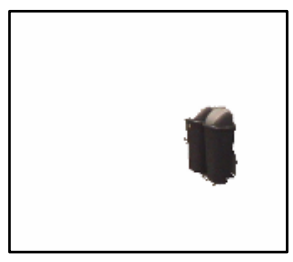

Frame 1596

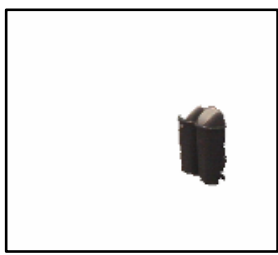

Frame 1597

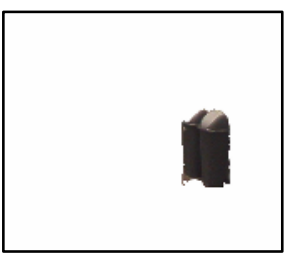

Frame 1598

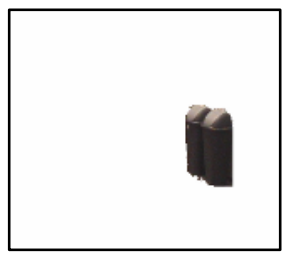

Fig. 6. Tracking of the wastebaskets on part of the original image sequence

\section{Conclusions and Future Work}

A dynamic iterative approach for object recognition and tracking in video sequences has been presented in which a probabilistic model at pixel level (or an approximation of it) is updated taking into account both the spot classification given by a trained neural net and prior object tracking information from the previous image. In this work, possibility images for each object of interest have been updated using a heuristic rule instead of applying a fully probabilistic model.

The use of the dynamic possibility images combined with the tracking information allows the gradual discrimination of the pixels classified as belonging to an object by the neural network but which do not really belong to it. It also helps to recover object pixels that have been classified as belonging to the background by the network but that really belong to an object. This can be made because the values in the possibility images save information of how the pixels have been classified in previous steps. Thus, this helps to decide at each iteration if a pixel belongs to an object or not. The experiments carried out have indicated that the proposed approach is viable and can provide satisfactory results.

In a future work, we would like to substitute the possibility images by actual probability images and to define the update and decision functions in a more principled way. We would also like to reduce the recognition and tracking computation time in order to approach the run-time processing. Our final objective is to design a robust dynamic approach to object recognition and tracking in video sequences based on unstructured sets of spots, which can deal with the variations in the object views resulting from the (relatively slow) motion of a mobile robot in an indoor environment.

\section{References}

1. Tu Z. Chen X., Yuille A.L., Zhu S.C., "Image Parsing: Unifying Segmentation, Detection, and Recognition", Proceedings in Ninth IEEE International Conference on Computer Vision, pp 18- 25 ISBN: 0-7695-1950-4 (2003).

2. Malik J., Belongie S., Leung T. and Shi J., "Contour and Texture Analysis for Image Segmentation", IJCV, vol.43, no.1, (2001).

3. Tu Z. and Zhu S.C., "Image segmentation by Data Driven Markov chain Monte Carlo", IEEE Trans. PAMI, vol. 24, no. 5, (2002). 
4. Zhu S.C. and Yuille A., "Region competition: unifying snakes, region growing, and Bayes/MDL for multiband image segmentation", IEEE Trans. on Pattern Analysis and Machine Intelligence, Vol: 18, Issue 9, pp:884 - 900 Sept. (1996).

5. Nelson, R.C.; Green I.A. "Tracking objects using recognition", Proceedings 16th International Conference on Pattern Recognition, ISSN: 1051-4651, ISBN: 0-7695-1695X Vol: 2, pp:1025-1030 (2002).

6. Yuya Iwasa, Ryuichi Oka "Spotting recognition and tracking of a deformable object in a time-varying image using two-dimensional continuous dynamic programming", CIT '04. The Fourth International Conference on Computer and Information Technology, ISBN: 07695-2216-5, pp:33 - 38. (2004).

7. Von Wichert G., "A probabilistic approach to simultaneous segmentation, object recognition, 3d localization, and tracking using stereo", in Lecture Notes in Computer Science 2191, pp 100, (2001).

8. Amézquita Gómez N. and Alquézar R. ,"Object Recognition in Indoor Video Sequences by Classifying Image Segmentation Regions Using Neural Networks “, Proceedings 10th Iberoamerican Congress on Pattern Recognition, CIARP 2005, LNCS Springer Berlin / Heidelberg ISSN: 0302-9743, Vol. 3773, Chapter: pp. 93 - 102 (2005).

9. Gerke, M, Heipke, C, Straub, B.-M. "Building extraction from aerial imagery using a generic scene model and invariant geometric moments", in Remote Sensing and Data Fusion over Urban Areas, IEEE/ISPRS Joint Workshop, ISBN: 0-7803-7059-7 8,pp: 8589, (2001).

10. Ballard D.H. and Brown C.M., Computer Vision, Prentice Hall, New Jersey (1982).

11. Serratosa F., Amézquita Gómez N. and Alquézar R. "Combining neural networks and clustering techniques for object recognition in indoor video sequences", Proceedings 11th Iberoamerican Congress on Pattern Recognition, CIARP 2006, Cancun, Mexico (2006). 